\title{
High Reproduction Rate versus Sexual Fidelity
}

\author{
A.O. Sousa and S. Moss de Oliveira \\ Instituto de Física, Universidade Federal Fluminense \\ Av. Litorânea s/n, Boa Viagem, Niterói 24210-340, RJ, Brazil
}

\begin{abstract}
We introduce fidelity into the bit-string Penna model for biological ageing and study the advantage of this fidelity when it produces a higher survival probability of the offspring due to paternal care. We attribute a lower reproduction rate to the faithful males but a higher death probability to the offspring of non-faithful males that abandon the pups to mate other females. The fidelity is considered as a genetic trait which is transmitted to the male offspring (with or without error). We show that nature may prefer a lower reproduction rate to warrant the survival of the offspring already born.
\end{abstract}

\section{Introduction}

It is not difficult to find in the animal kingdom species that live and work in sexual pairs, but sometimes have extra-pair relations. Biologists believe that these pairs are formed in order to better take care of the pups, and that the extra-pair relations have the genetic purpose to maximize the variability of their offspring or to produce some fitness benefit for them [1]. The Scandinavian great reed warbler is one of the species that presents these extra-pair matings. However, independent of its origin (social or genetic), true monogamy seems to be rare in Nature.

The Penna model for biological ageing [2] is a Monte Carlo simulation technique based on the mutation accumulation hypothesis. It has successfully reproduced many different characteristics of living species, as the catastrophic senescence of pacific salmon [3], the inheritance of longevity [4] and the self-organization of female menopause [5]. The extra-pair relations mentioned above have also been studied through this model [6]. Martins and Penna have obtained that the offspring generated by extra-pair relations are genetically stronger and present a higher survival probability than those generated by social relations. 
In this paper we are interested in using the Penna model to study the true monogamy, rarely found in Nature. One example is the California mouse. In this species a female is not able to sustain one to three pups alone. The pups are born at the coldest time of the year and depend on the parents body heat to survive. According to the biologist David Gubernick, as cited in Science [1], the situation is so dramatic that if the male leaves or is taken away, the female abandons or kills the pups. However, he also points out that other species of mice that live in the same environment are promiscuous. That is, the reason for true monogamy is still an open question under study. We have adopted the strategy of considering monogamy as a genetic trait, exclusively related to paternal care. Our assumption that male fidelity is genetically transmitted is analogous to the recent findings that the gene Mest regulates maternal care $[7,8]$.

In the next section we explain the Penna model and how fidelity is introduced. In section 3 we present our results and in section 4 the conclusions.

\section{The Sexual Penna model and Fidelity}

We will now describe the sexual version of the Penna model; details and applications can be found, for instance, in references [5,9]. The genome of each individual is represented by two bit-strings of 32 bits that are read in parallel; that is, there are 32 positions to be read, to each position corresponding two bits. One time-step corresponds to read one position of all the genomes. In this way, each individual can live at most for 32 time-steps ("years"). Genetic diseases are represented by bits 1. If an individual has two bits 1 (homozygotous) at the third position, for instance, it starts to suffer from a genetic disease at its third year of life. If it is an homozygotous position with two bits zero, no disease appears at that age. If the individual is heterozygotous in some position, it will get sick only if that position is a dominant one. The number of dominant genes and its randomly chosen positions are defined at the beginning of the simulation; they are the same for all individuals and remain constant. When the number of accumulated diseases of any individual reaches a threshold $T$, the individual dies.

The individuals may also be killed due to a lack of space and food, ac-

cording to the logistic Verhulst factor $V=1-N(t) / N_{\max }$, where $N(t)$ is the current population size and $N_{\max }$ the carrying capacity of the environment. 
At every time step and for each individual a random number between zero and 1 is generated and compared with $V$ : if this number is greater than $V$, the individual dies independently of its age or number of accumulated diseases.

If a female succeeds in surviving until the minimum reproduction age $R$, it generates, with probability $p, b$ offspring every year. The female randomly chooses a male to mate, the age of which must also be greater or equal to $R$. The offspring genome is constructed from the parents' ones; firstly the strings of the mother are randomly crossed, and a female gamete is produced. $M_{m}$ deleterious mutations are then randomly introduced. The same process occurs with the father's genome (with $M_{f}$ mutations), and the union of the two remaining gametes form the new genome. Deleterious mutation means that if the randomly chosen bit of the parent genome is equal to 1 , it remains 1 in the offspring genome, but if it is equal to zero in the parent genome, it is set to 1 in the baby genome. It is well known $[5,9,10]$ that due to the dynamics of the model, the bits 1 accumulate, after many generations, at the end part of the genomes, that is, after the minimum reproduction age $R$. For this reason ageing appears: the survival probabilities decrease with age. The sex of the baby is randomly chosen, each one with probability $50 \%$.

Let's see now how fidelity is introduced. We assume that if a female reproduces this year, she spents the next two following years without reproducing. So we consider two time steps as the parental care period. Remembering that in our simulations the female choses the male, if the male is a faithful one, he will refuse, during this period, to mate any female that eventually choses him as a partner. The non-faithful male accepts any invitation, but his offspring still under parental care pay the price for the abandonment: they have an extra probability $P_{d}$ of dying. The male offspring of a faithful father will also be faithful, with probability $P_{f}$. This means that if the father is faithful and $P_{f}=1$, the male offspring will necessarily be faithful. $P_{f}$ is also the probability of a non-faithful male having a non-faithful offspring.

\section{Results}

We start our simulations with half of the males faithful and half non-faithful. In Fig. 1 we show the final percentages (after many generations) of faithful males as a function of the offspring death probability $P_{d}$, for the cases 
where the male offspring inherits the father's fidelity state with probability $P_{f}=1$ (full line) and with probability $P_{f}=0.8$ (dashed line). This last case means that the offspring of a faithful father has a $20 \%$ probability of being non-faithful and vice-versa. From this figure we can see that as the death probability of the abandoned pups increases, the percentage of faithful fathers increases. From the solid curve it is easy to notice that there is a compromise between the lower reproduction rate of the faithful males and the death probability of the already born offspring abandoned by father: if $P_{d}<0.3$, a high reproduction rate dominates and after many generations the faithful males disappear from the population. However, for $P_{d}=1$ the opposite occurs, since there is a strong selection pressure against the non-faithful males to warrant the survival of the already born offspring.

From the dashed curve $\left(P_{f}=0.8\right)$ it can be seen that for $P_{d}=0$ a high percentage (greater than 20\%) of faithful males remains in the final population. The reason is that for $P_{d}=0$ there is no selection pressure. There is a probability that non-faithful males, which have a high reproduction rate, generate faithful offspring; these offspring are introduced into the population and, without any pressure, remain there. At this point $\left(P_{f}=0.8\right.$ and $\left.P_{d}=0\right)$ we have computed which percentages of faithful males descend from faithful and non-faithul fathers. We have obtained that for the $26.95 \%$ of faithful males that remain in the population, $9.28 \%$ of them descend from faithful fathers and $17.67 \%$ from non-faithful ones.

In Fig. 2 a we present the time evolution of the populations for $P_{f}=1$, and in Fig.2b for $P_{f}=0.8$. The inset show the final population sizes as a function of $P_{d}$. From Fig.2a it can be seen that the population sizes decrease until $P_{d}=0.4$ and then increase for increasing values of $P_{d}$, stabilizing around the same population size of $P_{d}=0.3$. For $P_{f}=0.8$ (Fig.2b) the population sizes decrease until $P_{d}=0.7$, and then stabilize around the same final size for increasing values of $P_{d}$.

Fig.3 shows the survival rates for $P_{f}=1$ and $P_{d}=0.0$ (circles), 0.5 (squares) and 0.9 (triangles). It can be noticed that for $P_{d}=0.5$ the child mortality is greater, since $P_{d}$ is already large and nearly $50 \%$ of the males (see fig.1, solid curve) are not faithful. The results obtained for $P_{f}=0.8$ are similar.

The survival rate is defined, for a stable population, as the ratio

$$
S(a)=N(a+1) / N(a)
$$


where $N(a)$ is the number of individuals of age $a$. A stable population means that the number of individuals of any given age $a$ is constant in time. It is important to emphasize that all curves presented here correspond to already stable situations. To obtain each of them we simulated 20 different populations (samples) during 800,000 time steps, and averaged the final results. The parameters of the simulations are:

Initial population $=20,000$ individuals (half for each sex);

Maximum population size $N_{\max }=200,000$;

Limit number of allowed diseases $T=3$;

Minimum reproduction age $R=10$;

Probability to give birth $p=0.5$;

Number of offspring $b=2$;

Number of mutations at birth $M_{m}=M_{f}=1$;

Number of dominant positions $=6$ (in 32).

\section{Conclusions}

We have used the Penna bit-string model for biological ageing to study the problem of true monogamy, rarely found in Nature. In our simulations a female that gives birth necessarily waits two time steps before giving birth

again. We call this period the parental care period. A faithful father also cannot reproduce during this period, but a non-faithful one can accept any female that randomly choses him to mate, abandoning the pups already born. The abandoned pups have, as a consequence, an extra probability to die. In this way there is a competition between the reproduction rate and the death probability of already born pups. We show that depending on this death probability, nature may prefers a lower reproduction rate to warrant the survival of those babies already born. We consider the paternal fidelity an expression of paternal care, and so admit it as a genetic trait to be transmitted to the male offspring.

Acknowledgements: to P.M.C. de Oliveira and D. Stauffer for important discussions and a critical reading of the manuscript; to CNPq, CAPES and FAPERJ for financial support. 


\section{References}

1- V. Morell, Science 281, 1983 (1998).

2- T.J.P. Penna, J.Stat.Phys. 78, 1629 (1995).

3- K.W. Wachter and C.E. Finch, Betwee Zeus and the Salmon. The Biodemography of Longevity, National Academy Press, Washington D.C.; T.J.P. Penna, S. Moss de Oliveira and D. Stauffer, Phys.Rev. E52, 3309 (1995).

4- P.M.C. de Oliveira, S. Moss de Oliveira, A.T. Bernardes and D. Stauffer, Lancet 352, 911 (1998).

5- S. Moss de Oliveira, P.M.C. de Oliveira and D. Stauffer, Evolution, Money, War and Computers, Teubner, Sttutgart-Leipzig (1999).

6- S.G.F. Martins and T.J.P. Penna, Int.J.Mod.Phys. C9, 491 (1998).

7- L. Lefebvre, S. Viville, S. C. Barton, F. Ishino, E.B. Keverne and M.A. Surani, Nature Genetics 20, 163 (1998).

8- R.S. Bridges, Nature Genetics 20, 108 (1998).

9- A.T. Bernardes, Annual Reviews of Computational Physics IV, edited by D. Stauffer, World Scientific, Singapore (1996).

10- J.S. Sa Martins and S. Moss de Oliveira, Int.J.Mod.Phys. C9, 421 (1998). 


\section{Figure Captions}

Fig.1 - Final percentages of faithful males in the population as a function of the death probability of the abandoned pups. The solid line corresponds to the cases where the offspring fidelity state is the same of the father. The dashed line corresponds to the cases where the offspring inherit the same fidelity of the father with probability $80 \%$.

Fig.2a - Time evolution of the populations (linear-log scale) for $P_{f}=1$ and different offspring death probabilities $P_{d}$. The inset shows the final population sizes as a function of $P_{d}$. For $0.6 \leq P_{d} \leq 1$ the final sizes are all very close to that for $P_{d}=0.3$.

Fig.2b - The same as Fig.2a for $P_{f}=0.8$.

Fig.3 - Survival rates as a function of age for $P_{f}=1$ and different values of $P_{d}$; circles correspond to $P_{d}=0.1$, squares to 0.5 and triangles to 0.9 . A higher child mortality can be noticed for $P_{d}=0.5$. 


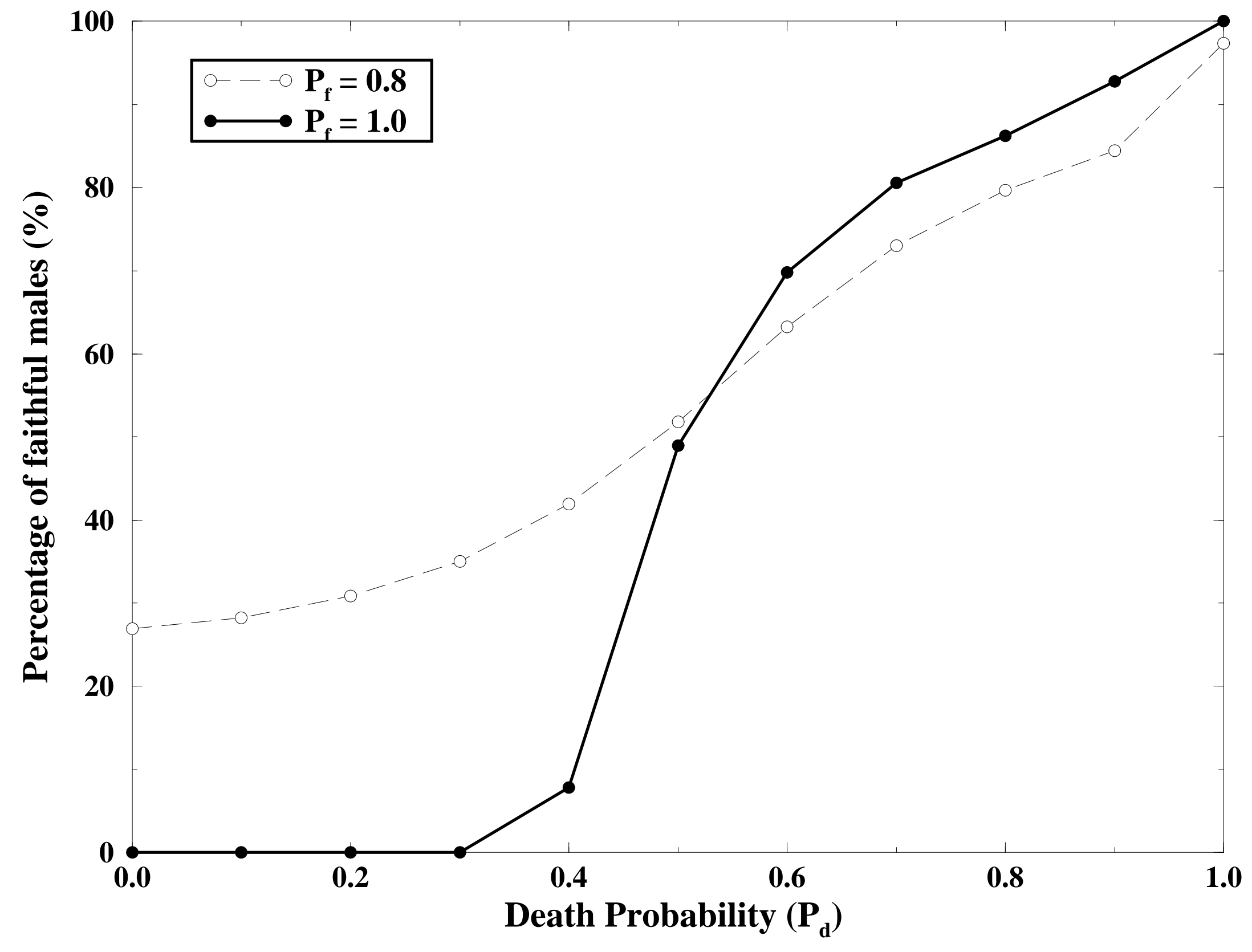




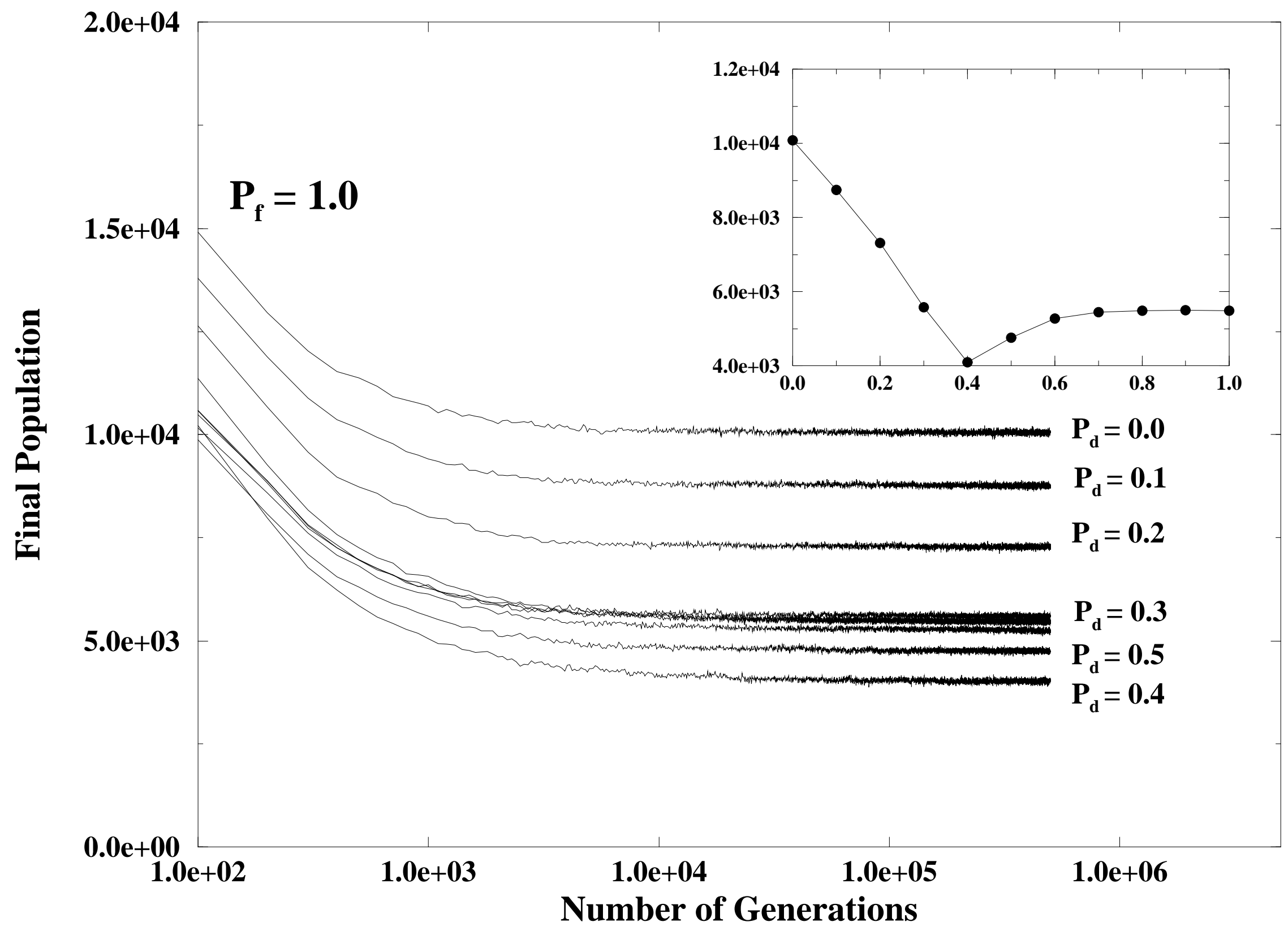




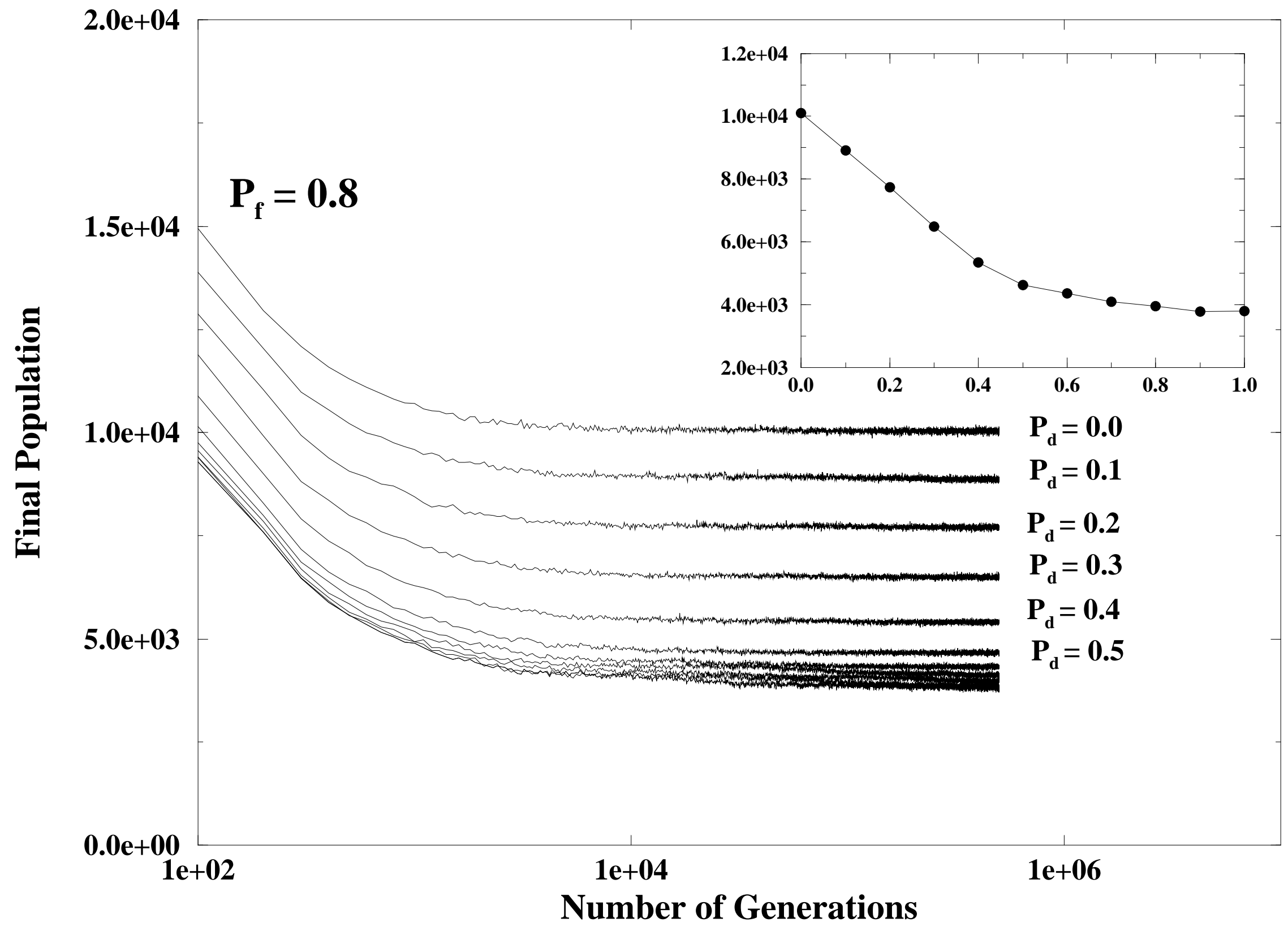




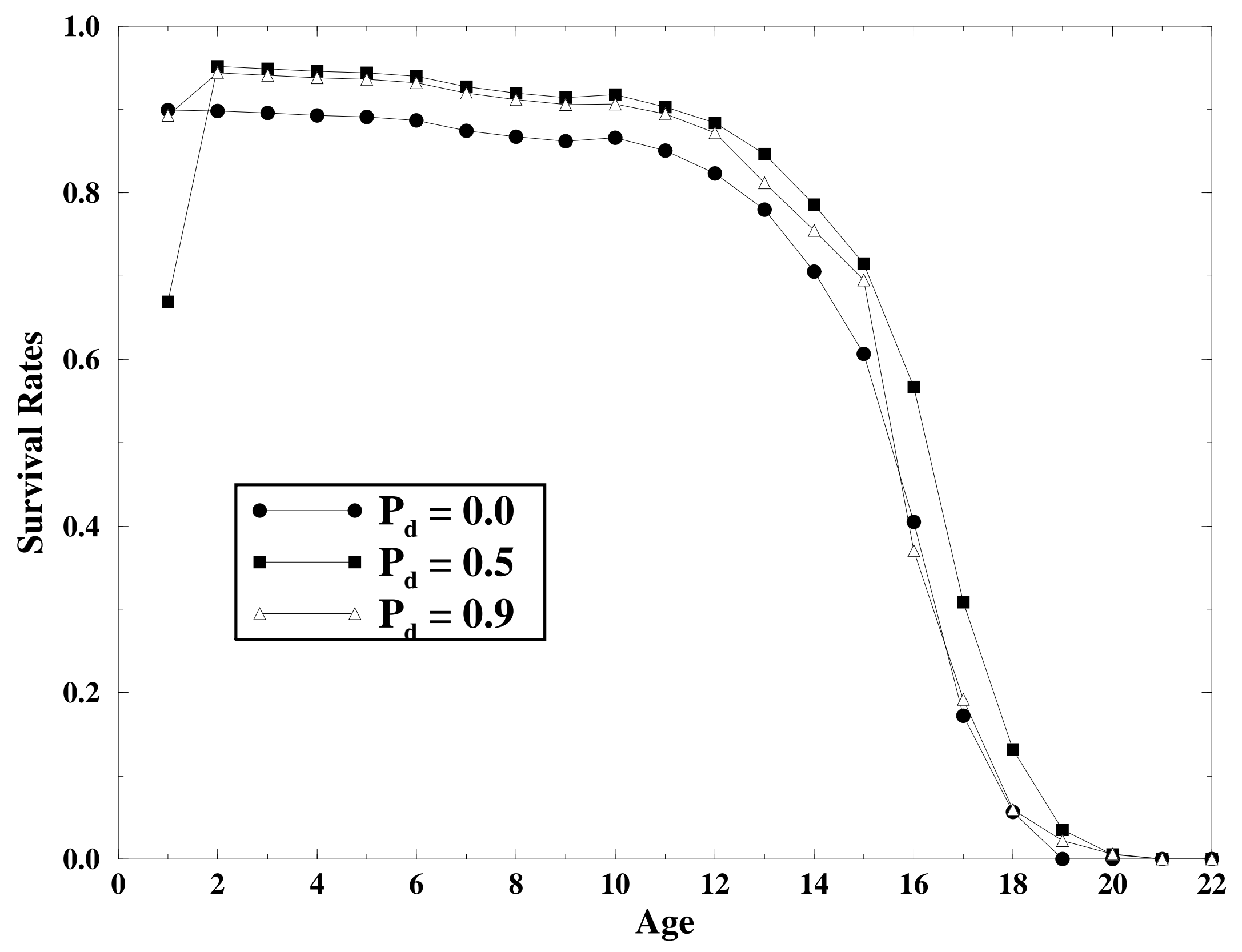

